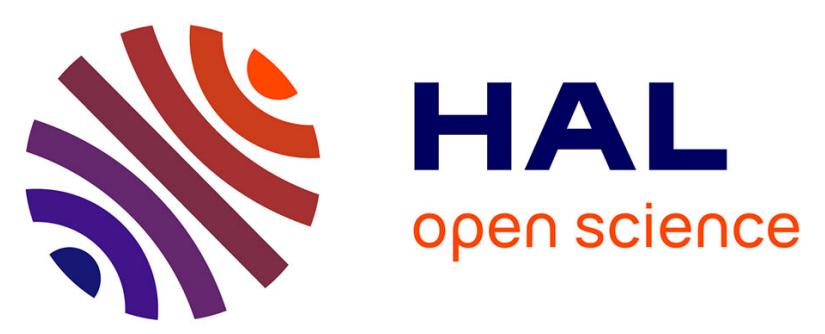

\title{
Des conseils citoyens inadaptés à la participation des jeunes? Retour sur trois expérimentations dans des quartiers populaires de la périphérie lyonnaise
}

\author{
Alice Daquin, Marine Huet, Emmanuel Martinais, Camille Martinez
}

\section{To cite this version:}

Alice Daquin, Marine Huet, Emmanuel Martinais, Camille Martinez. Des conseils citoyens inadaptés à la participation des jeunes? Retour sur trois expérimentations dans des quartiers populaires de la périphérie lyonnaise. Participations - Revue de sciences sociales sur la démocratie et la citoyenneté, 2019, 24, pp.83-109. hal-02308545

HAL Id: hal-02308545

https://hal.science/hal-02308545

Submitted on 8 Oct 2019

HAL is a multi-disciplinary open access archive for the deposit and dissemination of scientific research documents, whether they are published or not. The documents may come from teaching and research institutions in France or abroad, or from public or private research centers.
L'archive ouverte pluridisciplinaire HAL, est destinée au dépôt et à la diffusion de documents scientifiques de niveau recherche, publiés ou non, émanant des établissements d'enseignement et de recherche français ou étrangers, des laboratoires publics ou privés. 


\title{
Des conseils citoyens inadaptés à la participation des jeunes? Retour sur trois expérimentations dans des quartiers populaires de la périphérie lyonnaise
}

Pour citer cet article : Daquin A., Huet M., Lebian J., Martinais E., Martinez C., 2019, « Des conseils citoyens inadaptés à la participation des jeunes ? Retour sur trois expérimentations dans des quartiers populaires de la périphérie lyonnaise », Participations, n ${ }^{\circ}$, p. 83-109.

\section{Résumé}

Cet article s'intéresse à la création des conseils citoyens sous l'angle de la participation des jeunes des quartiers populaires. A partir d'expérimentations pédagogiques réalisées sur trois terrains de l'agglomération lyonnaise (Saint-Fons, Vaulx-en-Velin et Grigny), il interroge la capacité de ces nouveaux dispositifs participatifs à intégrer des publics de jeunes qui peinent en général à se mobiliser sur les enjeux de la cité. Bien que conçus selon des modalités pratiques différentes, ces trois supports d'analyse permettent néanmoins de mettre en forme deux résultats principaux. Le premier est que la mobilisation des jeunes, même souhaitée, reste contrainte par un ensemble de fragilités communes qui se manifestent dans des trajectoires scolaires et socio-professionnelles mouvementées, un rapport distancié et méfiant envers les institutions locales et un profond sentiment d'illégitimité vis-à-vis de la participation citoyenne. Le second est que la norme participative associée au fonctionnement des conseils citoyens s'ajuste mal aux attentes de ces publics et contribue, dans ces conditions, à reproduire les mécanismes qui détournent habituellement les jeunes des quartiers populaires des dispositifs de participation institutionnalisés.

\section{Mots-clés}

Conseil citoyen, participation des jeunes, quartiers populaires, politique de la ville, expérimentations pédagogiques

\begin{abstract}
This article analyses the creation of citizen councils under the perspective of youth participation in working class neighborhoods based on educational experiments that took place on three research fields in the urban areas of Lyon in France (Saint-Fons, Vaulx-en-Velin and Grigny). It questions the ability of this new participatory system to integrate young people who usually struggle to get involved in city stakes. Although conceived on different practical modalities, these three experiments bring out two major results. Firstly, the mobilization of the youth, although wanted, stays constrained by a set of common fragilities which occur in complicated socio-professional trajectories, a distant and distrustful relationship to local institutions and a deep feeling of illegitimacy concerning citizen participation. Secondly, the participatory norm related to the functioning of citizen councils has not adjusted well to the expectations of the public. It contributes, under these circumstances, to reproducing the mechanisms which usually turn away young people of working class neighborhoods from institutionalized participation.
\end{abstract}

\section{Keywords}

Citizens' council, participation of young people, popular district, policy for towns, teaching experiment 
Un rapide état des lieux des recherches consacrées à la participation des jeunes en France fournit deux types de constats. Premièrement, la plupart de ces travaux s'accorde pour montrer que les dispositifs destinés à renforcer l'inscription des jeunes dans l'espace public (conseils locaux de la jeunesse, parlements des jeunes, forums et autres budgets participatifs des lycées) ont surtout une visée d'éducation civique et d'intégration sociale par l'apprentissage de règles démocratiques de débat et de fonctionnement collectif (Becquet, 2006 ; Mazeaud, 2012). A ces enjeux d'inclusion, s'ajoutent aussi des objectifs politiques, la création des conseils municipaux de la jeunesse correspondant souvent à des tentatives d'élus locaux pour élargir leur espace de légitimité en dehors du système de vote, tout en ayant pour effet paradoxal d'isoler les jeunes du reste du jeu politique et notamment des vrais espaces de décision (Koebel, 2001). Les dispositifs participatifs dédiés à la jeunesse constituent ainsi rarement des tentatives d'implication des jeunes dans les décisions publiques qui les concernent en tant qu'habitants ou usagers (Loncle, 2008 ; Bataille, 2013). Deuxièmement, ces mêmes travaux signalent que les dispositifs participatifs plus généralistes (procédures de débat public, conseils de quartier, conseils de développement, budgets participatifs des communes, etc.) échouent la plupart du temps à attirer et tirer parti de la jeunesse habitante (Vulbeau, 2001). En effet, la place des jeunes dans ces dispositifs est rarement pensée, que ce soit a priori par leurs initiateurs publics ou a posteriori par leurs membres habitants. La spécificité des difficultés quotidiennes, des besoins, des temporalités et des capacités d'imagination, d'expression et d'action des jeunes du territoire restent ainsi trop peu posée (Hbila, 2014). Par conséquent, ces dispositifs, en reproduisant des formes et des modalités d'expression inadaptées aux jeunes, contribuent à perpétuer leur exclusion des espaces participatifs institutionnels.

Ce double constat, qui concerne tous les types de jeunes dans leur rapport à l'action publique, est encore plus sévère pour les jeunes des quartiers populaires, en particulier dans les domaines de la politique de la ville et de la rénovation urbaine où leur engagement est souvent requis (Tourette, 2006). Confrontées à cette difficulté, les institutions locales ont alors tenté, avec plus ou moins de succès (Hadj Belgacem, 2016), de retisser des liens entre cette jeunesse en voie de désaffiliation (ou jugée telle) et les espaces de représentation civique et politique qui régissent le fonctionnement des quartiers prioritaires de la politique de la ville (QPV). Les conseils citoyen $(\mathrm{CC})$, institués par la loi de programmation pour la ville et la cohésion urbaine du 24 février 2014, font partie de ces dispositifs de réaffiliation qui visent, depuis quelques années, à remettre les jeunes de ces quartiers dans une logique d'empowerment. Une des ambitions affichées de ces nouveaux outils participatifs est en effet de toucher les personnes et les collectifs les plus éloignés des institutions locales, en particulier les jeunes ${ }^{1}$. De ce point de vue, l'enjeu est de dépasser la conception étroite du « jeune de banlieue » comme menace ou victime à protéger (de la drogue, de la délinquance, du chômage, de la radicalisation), pour valoriser une conception plus positive du jeune comme ressource (Bordes, Vulbeau, 2004 ; Bordet, 2007).

L'objet de cet article est de s'intéresser à la création des CC sous cet angle particulier du recours à la jeunesse comme ressource : dans quelle mesure leur mise en place permet-elle de dépasser les limites de la mobilisation des jeunes des quartiers populaires pour les associer aux décisions qui les concernent directement, en tant qu'habitants, usagers ou simples citoyens ? Qui sont les jeunes visés par ces assemblées d'habitants et d'acteurs locaux, supposées contribuer de façon indépendante à l'élaboration et la mise en œuvre des contrats de ville ? Comment s'y prennent-

\footnotetext{
Sur ce point, voir notamment le rapport sur la citoyenneté et le pouvoir d'agir de Marie-Hélène Bacqué et Mohamed Mechmache qui a en partie inspiré la loi Lamy de février 2014 (Bacqué, Mechmache, 2013), ainsi que le Cadre de référence des CC de juin 2014 qui fixe les règles de mise en œuvre de ces outils participatifs.
} 
elles pour mobiliser ces publics souvent très éloignés des institutions locales et jusqu'à quel point réussissent-elles dans cette entreprise ?

Pour répondre à ces différentes questions, nous mobilisons trois expérimentations qui ont conduit, entre 2015 et 2017, des étudiants de niveau M2 à solliciter des jeunes de QPV de trois communes de la périphérie lyonnaise (Grigny, Vaulx-en-Velin et Saint-Fons) pour les préparer à une possible intégration au $\mathrm{CC}$ de leur quartier ${ }^{2}$. Conçues comme des exercices pédagogiques destinés à sensibiliser les étudiants aux enjeux et difficultés de la participation citoyenne, ces expérimentations ont fourni un important matériau de comptes rendus, d'observations et d'entretiens qui peut être utilement mobilisé pour documenter la création des CC sous l'angle de la participation des jeunes. Après avoir présenté nos supports d'analyse et la façon dont ils ont été négociés avec les acteurs locaux de la politique de la ville, nous verrons que cette intégration reste problématique et que la médiation des étudiants, même si elle permet de rassurer les jeunes sur leur capacité à participer, ne suffit pas à les décider de tenter l'aventure du CC. Nous verrons ensuite que si quelques-uns se laissent malgré tout convaincre, leurs rencontres avec les conseillers citoyens en place se soldent toutes par un échec. La chronique de ces rendez-vous manqués nous permettra enfin de montrer que, dans les cas étudiés, les CC reproduisent, voire renforcent, les mécanismes qui détournent habituellement les jeunes des dispositifs de participation institutionnalisés. A ce titre, ils ne seraient donc pas la solution attendue pour « retisser les liens », mais plutôt un obstacle supplémentaire entre les jeunes des QPV et les institutions qui régissent leur quotidien.

\section{Trois expériences pédagogiques visant l'intégration de jeunes dans les conseils citoyens}

Les analyses proposées ici ne sont pas fondées sur une enquête classique, mais sur un ensemble d'observations et d'entretiens réalisés dans un cadre pédagogique par des étudiants et leurs encadrants ${ }^{3}$. Bien que conçues selon des modalités pratiques différentes, ces trois démarches reposent sur un même parti pris: un cadre d'intervention négocié avec les acteurs institutionnels en charge de la création des $\mathrm{CC}$ et des étudiants invités à jouer un rôle d'intermédiaire entre d'un côté, des assemblées en cours de constitution désireuses d'attirer des «jeunes» et de l'autre, des groupes de jeunes susceptibles d'être intéressés par cette proposition ${ }^{4}$.

\footnotetext{
${ }^{2}$ Deux de ces expérimentations ont par ailleurs pris place dans le programme de co-évaluation présenté en introduction de ce numéro.

${ }^{3}$ Les étudiants mis à contribution sont issus de deux formations en urbanisme et aménagement: le master Altervilles de l'Université de Lyon d'une part, la voie d'approfondissement aménagement et politiques urbaines de l'École nationale des Travaux Publics de l'État (ENTPE) d'autre part.

${ }^{4}$ Précisons que les étudiants ne sont pas formellement missionnés pour jouer les entremetteurs entre les jeunes et les CC. Sur les terrains de Saint-Fons et Vaulx-en-Velin notamment, la commande vise surtout un objectif pédagogique. Formulée par les encadrants et non par les acteurs locaux, elle doit permettre aux étudiants de faire l'expérience d'une situation réelle de travail qui les intéresse en tant que futurs aménageurs et, par la même occasion, d'une relation d'échange avec un public proche géographiquement mais distant socialement. Car l'école d'ingénieur a beau être située en plein QPV, les étudiants ne sont pas vraiment intégrés à la vie du quartier. La plupart habite Vaulx-en-Velin pendant les trois ans de leur formation, mais très peu s'engagent localement et rares sont ceux qui nouent des relations avec les populations résidentes. Les activités sportives et la vie universitaire sur le campus ont en effet tendance à les isoler dans un entre-soi étudiant peu ouvert sur le monde extérieur. Pour les étudiants, le cours est donc l'objet d'une double découverte : de la participation en acte d'une part, des publics participants d'autre part.
} 


\section{Des assemblées en quête de jeunes qui échouent à recruter dans cette classe d'âge}

Lorsque nous les abordons, les CC partagent deux caractéristiques communes: ils sont disposés à s'intéresser à la question des jeunes et, à ce titre, aimeraient leur faire une place aux côtés des autres catégories de population plus faciles à représenter; ils n'ont pas réussi à suffisamment intéresser ce public pour le convaincre de s'intégrer au dispositif. Sur les trois terrains, cet intérêt pour la jeunesse et ses représentants recouvre en fait deux dimensions complémentaires. Il témoigne en premier lieu de l'adhésion des acteurs de la politique de la ville à la « règle du jeu » définie par le cadre de référence, notamment la préconisation stipulant que le collège des habitants doit «permettre de donner une plus grande place aux jeunes et renforcer ainsi leur participation à la vie démocratiques ». Cette volonté de se conformer à la règle ressort très nettement à Vaulx-en-Velin, où l'équipe du Grand Projet de Ville (GPV) chargée de créer le CC prévoit d'abord de réserver six places aux jeunes de 16 à 25 ans résidant dans les QPV, puis après un démarchage efficace auprès des deux lycées et de la mission locale ${ }^{6}$ de la ville, décide finalement de tirer au sort onze volontaires parmi une vingtaine de candidatures. Dans cette commune, la composition arrêtée par le préfet en juillet 2016 comprend donc un cinquième de jeunes, aux côtés d'une vingtaine d'habitants également tirés au sort, d'une douzaine de membres issus des conseils de quartier et de la même proportion d'acteurs économiques et associatifs, soit une structuration en classes d'âge assez conforme à celles des QPV de référence (où la proportion de moins de 25 ans oscille entre 35 et $45 \%$ ).

Cette quête des moins de 25 ans témoigne en second lieu de l'intention des trois CC d'investir le thème de la jeunesse et, dans ces conditions, de mobiliser les jeunes comme des ressources utiles, sinon indispensables, à la définition d'actions pertinentes, efficaces et adaptées aux réalités locales. La volonté de s'appuyer sur les jeunes pour appréhender et résoudre les problèmes qu'ils posent et/ou rencontrent collectivement s'affirme notamment à Saint-Fons où le sujet fait partie des priorités du contrat de ville'. L'intention de travailler pour et avec les jeunes en difficulté (ou supposés tels) se retrouve également à Grigny, où les membres du CC proposent dès les premières rencontres de s'attaquer aux occupations en pied d'immeuble considérées comme une forme de sociabilité juvénile problématique et une source de nuisances pour les habitants du quartier. L'une des participantes parmi les plus investies à la création du dispositif explique ainsi :

« Je voulais qu'on essaye d'associer les ados, car je trouve que les ados sont livrés à eux-mêmes. Ils emmerdent tout le monde... Enfin, ils emmerdent entre guillemets, car ils sont bien obligés de se mettre quelque part. Donc ils se sont retrouvés là-bas. Ça a fait fuir tous les gens d'une allée quand même. Il n'y a plus qu'un seul habitant dans l'allée 1. Donc ce n'est pas normal. Alors j'ai essayé de les associer à certains projets. Je suis allée les voir directement. Je leur ai parlé, je leur ai demandé ce qu'ils veulent et bien sûr, ils ont dit une salle pour eux, avec une console et machin. Moi, j'ai dit non: "Si on vous met une salle à disposition, ce n'est pas que pour la console. On n'a pas les moyens de l'acheter déjà. Il faut que vous ayez des projets". » (entretien)

\footnotetext{
${ }^{5}$ D'après le Cadre de référence des CC élaboré et diffusé en juin 2014 par le ministère des droits des femmes, de la ville, de la jeunesse et des sports (p. 6).

${ }^{6}$ La Mission locale est une association à but non lucratif œuvrant pour l'insertion sur le marché du travail de jeunes sans diplôme.

Cf. la convention locale d'application pour Saint-Fons du contrat de ville de la Métropole de Lyon 2015-2010 (p. 67).
} 
Conçue dans une logique thérapeutique par cette conseillère citoyenne, la participation des « jeunes hommes de l'allée $1^{8}$ » doit permettre de résoudre les problèmes posés par leur désœuvrement et leur présence continue au cœur du quartier. Seulement, la vision de ces jeunes comme un groupe social homogène et unifié capable d'agir dans le sens de l'intérêt commun, c'est-à-dire d'avoir des projets collectifs à même de les normaliser socialement, s'oppose au désir immédiat des individus sollicités (disposer d'un local pour jouer aux jeux vidéo) et empêche de facto leur intégration immédiate au dispositif. A Grigny, cette conception de la jeunesse en décalage avec les réalités locales n'est cependant pas la seule responsable de l'absence de représentants jeunes dans la composition du CC finalement arrêtée en mai 2016. L'incapacité de ses créateurs à mobiliser dans cette classe d'âge est aussi le fait d'un mode de recrutement par cooptation qui n'a pas vraiment permis d'atteindre ce public autrement que par l'intervention de la participante précédemment citée (Daquin, 2017b). Confiée au centre social de la commune, la mise en place du CC est en effet réalisée par une animatrice bien implantée dans le quartier prioritaire concerné (Le Vallon) et disposant d'un certain «capital d'autochtonie » pour y avoir habité dans les années 1980. Les personnes qu'elle convainc de tenter l'aventure du CC sont donc principalement des habitants qu'elle connaît bien, majoritairement des femmes de son âge (la cinquantaine) qu'elle a l'habitude de côtoyer dans les activités du centre social. Les plus jeunes, qui n'appartiennent pas à ce milieu d'interconnaissance, sont alors délaissés, au même titre que les établissements scolaires ou les associations en lien avec la jeunesse (amicale laïque, éducateurs spécialisés, clubs sportifs, mission locale). La ressource essentielle que constitue ces réseaux d'acteurs en prise avec les jeunes du quartier, en restant largement sous-exploitée, n'a donc pas permis d'atteindre cette catégorie de population. De même, le tirage au sort dans les listes de locataires fournies par les bailleurs sociaux agit comme un facteur discriminant pour les jeunes. A Grigny, comme à Saint-Fons où ce principe est également retenu pour susciter des candidatures, l'opération ne permet de contacter que les personnes inscrites sur les baux, c'est-à-dire les parents plutôt que leurs enfants, y compris les majeurs qui n'ont pas encore décohabité.

Dans les trois communes, le désir de jeunes ne suffit donc pas à mobiliser dans cette classe d'âge, même lorsque des démarches spécifiques ont été entreprises comme à Grigny (vers les jeunes hommes de l'allée 1) ou à Vaulx-en-Velin (en direction des lycéens et usagers de la mission locale). Dans ce dernier cas en effet, le succès initial ne se confirme pas puisque sur les onze volontaires initialement retenus par le GPV, seule une lycéenne se présente à la première réunion de juin 2016, puis à la deuxième de la rentrée de septembre, avant d'abandonner définitivement la place qui lui avait été attribuée. Notons toutefois que dans les trois cas, cette difficulté à recruter n'est pas spécifique aux jeunes, mais concerne également, quoiqu'à un degré moindre, les autres catégories de représentants ( $c f$. encadré à suivre)

Les trois CC ont en commun d'avoir eu beaucoup de mal à se constituer et à fidéliser leurs membres dans la durée. Lancés chacun avec une dizaine de conseillers habitants, ils ont ensuite vu leurs assemblées se réduire progressivement pour se stabiliser autour de cinq participants non institutionnels. Tous les trois ont été confrontés aux limites du tirage au sort, puisqu'aucun des membres réguliers n'a été recruté par ce procédé. A Grigny, c'est grâce au bouche-à-oreille que la mobilisation a été la plus efficace. L'animatrice du centre social, particulièrement intégrée dans la vie du quartier, a, par des relations interpersonnelles, réussi à réunir l'équipe des onze membres qui apparaissent dans l'arrêté

\footnotetext{
${ }^{8}$ A Grigny, l'expression «jeunes de l'allée $1 »$ est utilisée par les habitants, les professionnels associatifs, les représentants de la Ville et les membres du CC pour désigner un groupe d'une quinzaine de jeunes hommes âgés de 16 à 30 ans qui se sont appropriés le pied et la cage d'escalier de l'immeuble du bailleur Alliade Habitat sise Allée 1 du QPV. Cette catégorisation indigène sera réutilisée tout au long de l'article pour désigner ce groupe.
} 
préfectoral de création. Ce type de recrutement engendre finalement une homogénéité des profils des participants : sur ces onze personnes, neuf sont des femmes d'une cinquantaine d'années, occupant des emplois peu qualifiés (agent d'entretien, aide à la personne, etc.), dont les origines (portugaise, française, algérienne, tunisienne) reflètent la diversité du quartier. Formé d'habitants sans engagement politique ou associatif, le conseil citoyen de Grigny se distingue de son homologue de Vaulx-en-Velin qui comprend surtout des acteurs locaux représentant des associations, centres sociaux et conseils de quartier. L'assemblée vaudaise, telle qu'observée par les étudiants de l'ENTPE, se compose d'une majorité de femmes (au deux-tiers), âgées de 30 à 60 ans, et plutôt d'origine maghrébine. Enfin, son fonctionnement est marqué par une forte emprise du GPV : ce sont deux de ses représentantes qui animent les réunions, orientent les discussions et organisent l'évolution du conseil citoyen vers sa prise d'autonomie. A Grigny et Saint-Fons, le poids des institutions locales est également important : dans un cas, c'est le centre social qui s'impose comme principal animateur du conseil; dans l'autre c'est une coalition formée d'une consultante, d'une association locale et du service politique de la ville qui œuvre à la création de l'instance.

\section{Des étudiants entremetteurs qui proposent d'aider à la mobilisation des jeunes}

Sur nos trois terrains, l'intervention des étudiants se situe au moment où les participants des $\mathrm{CC}$ nouvellement institués (ou en cours de constitution à Saint-Fons) font l'expérience de leur incapacité à intéresser le public des jeunes et par conséquent, à recruter des représentants assidus dans cette classe d'âge. La proposition pédagogique, discutée avec les équipes de praticiens chargées de mettre en place ces nouvelles instances et de les accompagner vers la prise d'autonomie, se présente alors comme une mission d'assistance destinée à résoudre ce problème spécifique de la participation des jeunes dans les QPV. Dans les trois cas, les étudiants interviennent en médiation entre les représentants du CC et des groupes de jeunes préalablement identifiés comme étant susceptibles de s'associer aux travaux de ce dernier.

S'agissant de Saint-Fons, la proposition pédagogique conduit une vingtaine d'élèvesingénieurs spécialisés en urbanisme et leurs deux encadrants à prendre part au travail institutionnel de préparation qui précède la mise en place du CC. Mis à contribution durant le dernier trimestre 2015, soit bien en amont de la création effective de l'instance (qui aura lieu six mois plus tard, en mai 2016), les étudiants interviennent en soutien du service politique de la ville qui prévoit de recruter les conseillers citoyens parmi 250 habitants tirés au sort dans les listes de locataires fournie par les bailleurs sociaux du quartier concerné. A mi-parcours, une journée de terrain est organisée au cours de laquelle les étudiants se chargent de prendre contact avec les habitants sélectionnés pour tester leur adhésion au projet et les convier à une réunion préparatoire à la création du conseil organisée par la ville. La difficulté de l'exercice et les échecs répétés devant des portes qui refusent de s'ouvrir (ou se referment trop vite) conduit cependant une partie du groupe d'étudiants-enquêteurs à détourner la commande et à profiter de leurs allées et venues sur le quartier pour solliciter plusieurs jeunes croisés au pied des immeubles, dont certains s'avèrent finalement beaucoup plus faciles d'accès que les habitants visés. Intéressés, ou plus sûrement curieux de la démarche, une partie de ces jeunes du quartier se laisse alors convaincre d'assister à la réunion préparatoire. Tenant parole, quelques-uns se présenteront le jour dit, retrouvant les étudiants alors chargés d'accueillir les participants par une restitution des résultats de leur enquête sur le quartier. Arrivant à son terme, le cours se

\footnotetext{
${ }^{9}$ Sur les trois terrains, on note que les étudiants jouent d'autant plus facilement ce rôle d'entremetteur qu'ils soutiennent globalement, sans vraiment l'interroger, l'idée du CC comme dispositif de restauration de la participation des jeunes des quartiers populaires. Dans ces conditions, le sens de leur intervention et de la commande à laquelle ils sont censés répondre font assez peu débat, entre eux et avec les encadrants.
} 
conclut fin 2015 par la rédaction d'un carnet de terrain comprenant les comptes rendus écrits de la quarantaine d'entretiens effectuée avec les habitants du quartier ainsi qu'une synthèse des observations réalisées lors de la réunion de préparation.

L'expérience de Saint-Fons ayant donné satisfaction au plan pédagogique, la décision est prise de la reconduire l'année suivante sur le terrain de Vaulx-en-Velin (commune d'implantation de l'ENTPE). La proposition, préalablement discutée avec l'équipe du GPV en charge du CC, est cependant revue pour s'ajuster à la situation locale et aux attentes de nos interlocuteurs. Deux objectifs spécifiques sont ainsi définis : 1) comprendre pourquoi les lycéens démarchés quelques mois plus tôt ont finalement décidé de ne pas participer ; 2) voir dans quelle mesure et à quelles conditions les volontaires retenus par le GPV et/ou tout ou partie de leurs camarades de classe, seraient finalement disposés à intégrer le CC. Répartis en deux équipes, les étudiants entrent alors en contact avec une classe de première commerce et une terminale littéraire de chacun des lycées de la ville, puis créent avec ces deux groupes de jeunes la possibilité d'un échange approfondi sur leurs expériences d'habitant, d'usager et de citoyen dans un quartier prioritaire de la proche banlieue lyonnaise. Plusieurs séances d'atelier, ponctuées de temps de restitution, permettent ainsi de capter leur vision des dispositifs participatifs en général et du $\mathrm{CC}$ en particulier, de les interroger sur les sujets qui mériteraient selon eux d'être pris en charge par une telle instance et, en même temps, de recueillir leurs idées pour encourager la participation des jeunes. Ces échanges, consignés dans deux carnets de terrain réalisés par les étudiants à l'issue du cours, donnent finalement lieu à une restitution auprès des membres du $\mathrm{CC}$ et de deux représentantes du GPV, en présence de cinq lycéens qui ont finalement accepté de tenter l'aventure.

L'expérience de Grigny enfin, bien qu'ayant lieu simultanément à celle de Vaulx-en-Velin, prend forme de façon indépendante à cette dernière, dans un contexte pédagogique différent. Sur ce troisième terrain, la proposition de médiation avec le CC émerge durant l'automne 2016, à l'occasion d'une enquête d'usages réalisée par une étudiante du master Altervilles de l'Université de Lyon dans le cadre de son stage à la direction de la politique de la ville de la Métropole de Lyon (Daquin, 2017b). Cherchant à comprendre le fonctionnement du quartier du Vallon et le rapport des habitants aux espaces extérieurs, celle-ci se retrouve rapidement confrontée au problème de l'allée 1 évoqué plus haut, c'est-à-dire à ces jeunes hommes de 16 à 30 ans environ qui depuis plusieurs années font territoire dans ce lieu en ayant progressivement fait fuir une partie des occupants de l'immeuble. Une restitution de l'enquête, en présence de membres du $\mathrm{CC}$ et de quatre jeunes de l'allée 1 visés par les récriminations habitantes crée alors les conditions d'un nouvel échange entre les deux parties qui se solde cette fois par une invitation ferme à se retrouver dans le CC pour discuter plus avant de la demande des jeunes. Bénéficiant d'un bon contact avec ces derniers, l'étudiante se propose de jouer les intermédiaires et profite de cette position privilégiée pour légitimer sa présence sur le terrain, réaliser l'enquête de son mémoire de M2 et s'engager en parallèle dans le processus de coévaluation des CC qui démarre au même moment (Daquin, 2017a). Cette double démarche de recherche, qui se prolonge jusqu'au printemps 2017, donne lieu à une quinzaine d'entretiens (dont une dizaine avec des jeunes du quartier et des membres du CC) et à l'observation de cinq réunions de l'instance de participation.

Rassemblées dans une même perspective analytique, ces trois expériences constituent finalement une série de points de vue sur la constitution des $\mathrm{CC}$, la façon dont ces groupes d'habitants envisagent de s'ouvrir aux jeunes et les liens qu'ils cherchent à nouer avec cette catégorie de population. Les observations menées montrent pourtant que les sollicitations s'adressent à des publics a priori différents : des jeunes adultes poursuivant leurs études ou en recherche d'emploi à Saint-Fons, des élèves de première et terminale des deux lycées de Vaulxen-Velin et enfin, des hommes entre 16 et 30 ans marqués par le désœuvrement et l'occupation 
de « l'allée 1 » de Grigny. Qui sont précisément ces jeunes convoités par les trois CC ? Sontils si différents les uns des autres ? Comment vivent-ils l'expérience participative proposée par les étudiants ? Comment se conclut, pour chacun d'eux, ces tentatives d'intégration? C'est à ces différentes questions que nous proposons de répondre maintenant.

\section{Des jeunes faiblement disposés à jouer les conseillers citoyens}

L'essai de caractérisation que nous proposons maintenant vise à reconnaître certains traits communs parmi les publics de jeunes convoités par chacun des trois CC étudiés. Les jeunes hommes de l'allée 1 de Grigny, les lycéens de Vaulx-en-Velin et les jeunes adultes plus indéfinis de Saint-Fons ne forment pas un ensemble homogène, loin de là : les individus qui composent ces groupes se différencient même assez facilement lorsqu'on les considère à partir de critères objectifs comme l'âge, le genre, le rapport à l'institution scolaire ou la profession des parents. Cependant, les investigations montrent qu'en dépit de leurs différences, ces jeunes présentent des caractéristiques assez semblables quand on les envisage dans leur rapport à la participation institutionnalisée.

\section{Les catégorisations de la jeunesse à l'œuvre}

La jeunesse n'est pas une catégorie stable et uniforme, mais une construction sociale qui mobilise l'ensemble des institutions susceptibles d'interagir avec elle (Tourillhes, 2006). La « jeunesse n'est qu'un mot», disait Bourdieu (1984) pour illustrer le fait que sa définition est l'objet de luttes permanentes. A leur niveau, les conseillers citoyens n'échappent pas à cette production de sens. Par leurs discours, leurs interventions et leur conception des problèmes, ils contribuent les uns les autres à dessiner les contours de ce fameux « jeune » qui leur fait tant défaut, que ce dernier prenne les atours de « l'adolescent de l'allée 1 », du « lycéen de quartier populaire », de « l'usager de la mission locale » ou de « l'oisif en difficulté d'insertion sociale et professionnelle ». Le cas de Grigny illustre bien cette idée en donnant à voir comment l'action du CC vient durcir la catégorie des «jeunes de l'allée 1 » et renforcer son caractère problématique vis-à-vis des institutions locales, du bailleur social et, plus largement, des habitants du quartier. Forgée il y a plusieurs années pour qualifier l'usage plus ou moins licite de l'immeuble du quartier communément désigné « l'allée 1 » par les jeunes eux-mêmes, cette catégorie ne revient pas par hasard dans les discussions du CC. La figure du « jeune squatteur à capuche en pied d'immeuble », souvent associée au trafic de drogue, à la délinquance ou aux violences urbaines (Mucchielli, 1999), fait en effet figure d'allégorie stigmatisante des quartiers de grands ensembles (Derville, 2017 ; Guénolé, 2015). En tant qu'habitants ou professionnels de la politique de la ville, les conseillers citoyens ne sont pas imperméables à cet imaginaire de la banlieue qu'ils convoquent d'autant plus facilement qu'il s'agit de qualifier et de comprendre les problèmes du quartier auxquels ils sont quotidiennement confrontés. Dans leurs propos, les occupants de l'allée 1 apparaissent ainsi sous les traits de cette « jeunesse en difficulté » propre aux quartiers populaires, c'est-à-dire des jeunes hommes livrés à euxmêmes, victimes de l'abandon des institutions locales en charge de la jeunesse, en particulier de la municipalité. C'est ce que confirme cette représentante des habitants en réponse à une question sur les aspects négatifs du quartier qui mériteraient selon elle d'être pris en compte par le CC :

«Ben le négatif... C'est ceux dont on ne s'occupe pas. C'est les jeunes. En fait, on s'occupe de la tranche de 3 à 14 ans. On prend quelques gamins entre 15 et 17 ans pour faire des travaux, pour leur faire gagner un peu de sous. Mais après, entre 18 et 25 ans... Il y en a qui vont à l'école, mais même ceux-là ils sont abandonnés. Cette tranche d'âge... Il n'y a rien qui leur est proposé. Alors c'est vrai qu'on dit qu'ils sont majeurs, qu'ils ont une voiture, qu'ils peuvent se débrouiller 
machin... Mais ils sont sans travail. Pareil, ceux qui sont encore à l'école n'ont pas de sous. J'aimerais bien qu'on trouve une solution pour ça. » (entretien)

Il est cependant important de noter que là où la plupart des institutions locales aurait sans doute mobilisé le registre de la «jeunesse en tant que menace », les membres du CC de Grigny se situent plutôt sur celui de la « jeunesse comme ressource » en ne désignant pas uniquement les jeunes de l'allée 1 comme cibles de leur intervention, mais également comme acteurs potentiels de leur réinscription sociale (Vulbeau, 2001). De fait, nombreux sont les habitants interrogés à les voir comme des ressources plutôt faciles d'accès, dans la mesure où ils sont toujours à proximité immédiate du local où se tiennent les réunions du $\mathrm{CC}$, qu'ils sont perçus comme n'ayant rien à faire (sinon des bêtises) et qu'ils se montrent plutôt réactifs en cas de sollicitation (notamment lorsqu'il s'agit d'organiser des événements festifs sur le quartier).

A Vaulx-en-Velin, le type de jeune convoité par le $\mathrm{CC}$ est également conçu en termes de ressources. Mais n'étant pas associé à un problème en particulier, il n'est pas envisagé selon des caractéristiques prédéfinies, sinon celle de résider dans l'un des QPV concernés et d'être toujours scolarisé ou engagé dans une démarche active de recherche d'emploi. En dirigeant les sollicitations vers les deux lycées de la ville et la mission locale, les membres du CC témoignent toutefois de leur volonté de favoriser une certaine diversité dans la représentation des jeunes. Celle-ci vise notamment l'âge des publics sollicités, qui correspond potentiellement à toutes les périodes de la jeunesse (Galland, 2009), ainsi que les parcours vers la vie active. Parmi les jeunes des quartiers défavorisés, la scolarité est un vecteur de différenciation important qui confère un statut d'élite aux lycéens des filières générales et technologiques tout en déqualifiant les décrocheurs mais également leurs homologues des filières professionnelles (Truong, 2015). A Vaulx-en-Velin, les jeunes scolarisés au lycée d'enseignement général se définissent ainsi en opposition à leurs pairs de l'enseignement professionnel et plus largement, aux « jeunes squatteurs à capuche en pied d'immeuble » qu'ils associent systématiquement à la figure péjorative des « racailles » (Huet, 2018). Dans la tête des jeunes des QPV rencontrés par les étudiants, ces catégories ont alors tendance à masquer le fait que la figure du «lycéen de banlieue » recouvre une grande diversité de parcours, de modes de vie et de rapport à la culture des rues (Lepoutre, 1997).

Ces deux exemples de Grigny et Vaulx-en-Velin montrent bien que le jeune issu des QPV constitue une catégorie aux contours labiles, dont le contenu dépend largement des attentes et mises en forme opérées par les CC, que cela soit par nécessité (répondre à un problème saillant vécu comme une nuisance), par praticité (entrer en contact avec un public d'accès facile) ou encore par effet de représentation (atteindre cette figure abstraite et convoitée du lycéen de quartier populaire).

\section{Sous les catégories, des situations assez comparables}

L'analyse des jeux «sur» et «avec » les catégories peut cependant être complétée par une étude des discours et des postures adoptées par les jeunes eux-mêmes. L'observation des échanges entre étudiants et lycéens de Vaulx-en-Velin et les entretiens réalisés avec les jeunes de Grigny et Saint-Fons, permettent alors de faire ressortir quatre traits communs à ces trois groupes : une grande vulnérabilité qui affaiblit leur capacité à se projeter dans l'avenir ; une connaissance quasi inexistante des moyens et des formes d'expression permettant d'intervenir dans la vie de la cité ; un sentiment dominant d'illégitimité face aux demandes de participation ; et enfin, des expériences de discrimination répétées qui génèrent résignation et perte de confiance dans les institutions.

Le premier trait commun est en rapport avec les caractéristiques sociales des populations vivant dans les QPV (Avenel, 2004). Comme l'ont déjà montré nombre de travaux de sociologie 
urbaine, la jeunesse des banlieues se définit par une certaine fragilité, dans la mesure où elle est plus que les autres touchée par les inégalités scolaires et le chômage ${ }^{10}$, qu'elle souffre d'un accès moindre à la mobilité et aux services publics et enfin, qu'elle est plus éloignée des formes classiques de la démocratie locale (INJEP, 2012). Ainsi, les jeunes rencontrés en entretien à Grigny sont tous d'origine maghrébine ou portugaise, témoignent de parcours scolaires et professionnels marqués par une entrée précoce dans la vie active (formations courtes ou déscolarisation après le collège), sortent rarement du quartier et se sentent globalement peu concernés par leurs devoirs de citoyen (s'agissant du vote notamment). De même, les lycéens de Vaulx-en-Velin sont très majoritairement d'origine étrangère (maghrébine et subsaharienne), vivent en moyenne dans des foyers à bas revenus et, d'après les témoignages consignés par les étudiants, se destinent majoritairement à des études courtes, dans des filières très appliquées". Même s'ils se distinguent par leurs parcours, les occupants de l'allée 1 de Grigny, les jeunes de Saint-Fons et les lycéens de Vaulx-en-Velin partagent donc un certain nombre de caractéristiques de cette jeunesse « plurielle et vulnérable » des quartiers populaires qui peine à s'inscrire dans une stabilité sociale et professionnelle et parvient difficilement, sinon pas du tout, à se projeter dans l'avenir (Hbila, 2014).

Le second trait commun des jeunes rencontrés par les étudiants concerne le rapport à l'action publique et à la démocratie locale. Revendiquant le fait de se tenir loin de la vie publique, la plupart n'est pas vraiment intéressée par l'action municipale. Contrairement à une partie de leurs aînés des années 1980 et 1990 (Lapeyronnie, 1987), peu sont politisés et aucun ne déclare d'activité militante. Le vote semble également faire problème, même aux élections municipales. Tous témoignent en fait de ces processus d'évitement du politique observés par ailleurs dans les associations locales de jeunes d'origine maghrébine (Hamidi, 2006) qui se caractérisent par une absence notable de « montée en généralité » et de « conflictualisation » à propos de leur statut précaire et plus largement, de leurs expériences quotidiennes en tant qu'habitant ou usager. Sur ce registre, on observe également que beaucoup ne savent pas vraiment définir la politique de la ville et que tous méconnaissent les dispositifs dont ils sont pourtant les cibles prioritaires (Estèbe, 2001), qu'il s'agisse d'insertion, de loisirs, de sport, de santé et de culture. Cela concerne aussi le CC, dont aucun n'avait entendu parler avant d'être sensibilisé à son fonctionnement par les étudiants. A l'instar de nombreux habitants et parfois même de certains professionnels, le sens de cette instance reste globalement difficile à saisir pour l'ensemble de ces jeunes.

Le troisième trait commun aux jeunes interrogés par les étudiants concerne justement leur mise à contribution dans un dispositif comme le CC. Invités à discuter de cette éventualité et à se projeter en tant que membre d'une telle assemblée, beaucoup commencent par se déclarer globalement incompétents pour ce genre d'exercice. Trois raisons semblent alimenter ce sentiment d'illégitimité : leur jeune âge et leur relative inexpérience qui ne leur paraissent pas correspondre aux besoins d'une telle institution; le faible intérêt qu'ils éprouvent pour leur cadre de vie, jugé sans attrait et enfin, la difficulté de bon nombre d'entre eux à se penser comme des citoyens de plein droit. Ce déficit d'accomplissement de la citoyenneté, caractéristique des populations d'origine étrangère des quartiers populaires (Castel, 2006), est

\footnotetext{
${ }^{10}$ Selon l'enquête Emploi de l'INSEE (Rapport 2012 de l'ONZUS), seuls 21,5\% des jeunes des quartiers populaires âgés de 15 à 24 ans sont actifs en 2011 (contre 28,4\% pour les unités urbaines environnantes). Pour le reste, $14,6 \%$ sont au chômage (contre 7,9 \% pour les unités urbaines environnantes), 52,7 \% sont en cours d'études ou formation (contre 58,3\% pour les unités urbaines environnantes) et $11,1 \%$ sont comptabilisés comme autres inactifs (INJEP, 2012).
}

"Ce que confirme l'enquête réalisée par Marine Huet dans le cadre de sa thèse sur les lycéens de Vaulx-en-Velin (Huet, 2018). 
particulièrement vif chez les jeunes de Grigny, ce que confirme cette éducatrice spécialisée en prise avec les jeunes de l'allée 1 :

\begin{abstract}
«Au niveau politique, on parle beaucoup de l'accès à la citoyenneté. Parce qu'en fait, ce sont des jeunes qui ne se sentent pas citoyen français. Ils se sentent immigrés, ils ne se sentent pas français. Pourtant on leur dit : "Vous avez une carte d'identité et cette carte d'identité vous donne le droit d'aller voter". C'est vraiment une histoire de ne pas se sentir français et ça, ça me questionne beaucoup. Alors quand je les connais bien... Quand je les connais peu, je ne me permets pas (rires)... Mais quand je les connais bien, je leur dis : "Vous avez une carte d'identité française, vous êtes français". Et puis ils ont des réflexions du genre : "Tiens, lui, il joue le français". Il y a plein d'expression comme ça, "Il fait le français", etc. » (entretien)
\end{abstract}

Chez beaucoup de jeunes interrogés, la stigmatisation médiatique et politique des QPV renforce ce phénomène de distinction entre la nationalité administrative et les représentations sociales collectives. La plupart a en effet tendance à catégoriser le monde social de manière dichotomique, en opposant la périphérie populaire des grands ensembles aux centres-villes des agglomérations auxquelles ils se rattachent. Le plus souvent, cette différenciation s'effectue sur des critères sociaux, ethno-raciaux et moraux. Les habitants des centres urbains sont alors perçus comme des bourgeois blancs, c'est-à-dire des «vrais français », tandis que les populations de Vaulx-en-Velin, de Grigny ou de Saint-Fons, sont principalement appréhendées par leurs origines maghrébines ou sub-sahariennes. Cette stigmatisation renforce alors la détresse sociale des jeunes en l'inscrivant dans une logique de discrimination négative dont ils peinent à se dépêtrer. La lassitude, voire l'exaspération que cela génère, expliquent le découragement que suscite l'idée de leur participation au CC et l'absence de motivation pour s'investir dans une démarche qui leur paraît sans enjeu.

A ce découragement, qui se dit souvent avec les deux mêmes mots (« la flemme »), s'ajoute enfin le sentiment d'injustice qui résulte des discriminations vécues par la plupart de ces jeunes, dans leurs relations à la police et la justice, pour accéder à l'emploi et s'y maintenir, ou pour décrocher un stage. Comme l'explique Régis Cortéséro, ce sentiment d'injustice décrit aujourd'hui une situation de mise à l'écart, de bannissement de la cité, qui opère comme un « substrat axiologique » à partir duquel les jeunes des banlieues appréhendent les dispositifs proposés par les institutions (Cortéséro, 2010). L'exemple de Kamel, un des jeunes de l'allée 1 de Grigny, illustre bien cette idée. Pour justifier son manque d'intérêt pour le CC, il convoque en effet son histoire familiale et l'exemple de son père, enrôlé de force par l'armée française en Algérie, mais jamais gratifié en retour pour ce service rendu à la nation. Dans son propos, le sentiment de non reconnaissance du sacrifice paternel, mais aussi la stigmatisation des « arabes » suite aux attentats terroristes de 2015, renforcent son impression d'être traité injustement par la puissance publique. Le sentiment qui domine est alors celui de l'impuissance et de la résignation, dissuadant le jeune de s'engager pour faire valoir sa voix politiquement sur les enjeux qui le concernent :

Étudiante : «Et aujourd'hui, si tu pouvais avoir le maire en face de toi et lui demander ce que tu veux, ce qui manque pour les jeunes, qu'est-ce que tu lui dirais? »

Kamel : « Aujourd'hui, mon temps il est passé. Moi ça y est, j'en ai plus rien à foutre. Je lui dirai que j'en ai rien à foutre de leurs politiques en vrai. Ils font comme ils veulent en vrai! Moi, je ne vais rien changer. Je peux que subir. Comme tout le monde quoi. » (entretien)

De même, les lycéens interrogés expriment volontiers leur méfiance face aux institutions et leur vision désabusée des procédures participatives : «c'est trop beau pour être vrai », dit l'un d'eux. Le CC est alors volontiers considéré comme une entité factice, un simulacre de 
participation citoyenne, «pour laisser croire aux gens qu'on va les écouter, sans jamais rien faire ${ }^{12} \gg$.

Ainsi, malgré une pluralité de trajectoires, les jeunes rencontrés présentent une série de traits communs qui nous conduit à les considérer comme faisant partie d'une même " communauté d'expérience » (Aquatias, 1997 ; Bier, 2009) ou « communauté de destin » (Marlière, 2007). Ces expériences comparables, qui alimentent un profond sentiment d'exclusion et d'illégitimité, agissent alors comme autant de freins qui, en dépit de la relation de confiance établie par les étudiants, les retiennent presque malgré eux de tenter l'aventure de la participation et de se risquer à pousser la porte des $\mathrm{CC}$.

\section{Des invitations à participer largement esquivées}

Même motivée par les étudiants, la participation des jeunes reste foncièrement problématique. Confrontés à l'invitation du CC, beaucoup réagissent par l'esquive, soit directement en refusant tout net la proposition, soit indirectement en laissant penser à leur motivation et en ne se présentant pas le jour dit. Sur la totalité des jeunes ainsi sollicités, seule une petite minorité surmonte ses appréhensions et ses réticences en passant concrètement à l'acte.

A Vaulx-en-Velin, si quinze lycéens sont partants pour une rencontre avec le CC à l'issue des ateliers menés par les étudiants, et malgré des rappels effectués par téléphone dans l'intervalle, seul un tiers est au rendez-vous fixé la semaine suivante. Les cinq jeunes à s'être finalement déplacés sont des Terminale $\mathrm{L}$ du lycée d'enseignement général. Invités à restituer le contenu des travaux menés avec les étudiants, ils passent alors en revue les raisons qui expliquent leur faible attirance pour le $\mathrm{CC}$, les motivations qui pourraient dans ces conditions les aider à participer et, enfin, les sujets qu'ils défendraient s'ils étaient conseillers citoyens. Très à l'écoute, les habitants présents à la réunion réagissent positivement à ces propositions, notamment celles consistant à revaloriser l'image de Vaulx-en-Velin et la place des femmes dans la ville. Ils rassurent ainsi les jeunes quant à l'intérêt de leur participation, avant de conclure l'échange en leur suggérant « de revenir pour travailler avec le CC sur la question de la restauration de l'image de la ville ». Mais malgré l'accord donné par deux d'entre eux, aucun ne se présentera à la réunion suivante, ni à celles d'après, faute d'avoir été plus clairement invité à prendre place dans l'assemblée et remobilisé par la suite. Dans ce cas précis, les freins à la participation évoqués plus haut semblent avoir joué à plein, incitant les jeunes présents à la réunion à privilégier l'exit plutôt que de prendre le risque d'un engagement jugé hasardeux.

A Saint-Fons, le résultat n'est guère plus satisfaisant pour les étudiants qui, lors de la journée passée sur le quartier, avaient pourtant convaincu quelques jeunes de participer à la réunion de préparation à l'installation du CC. Effectivement présents le jour dit, ces derniers se maintiennent cependant à l'écart du groupe principal des participants et peinent à s'intégrer à la discussion. Déroutés par le format de la réunion et peu intéressés par les sujets de discussion proposés par les animateurs, ils optent finalement pour l'esquive en quittant discrètement la réunion au bout d'une vingtaine de minutes. A Grigny en revanche, le seul jeune à se présenter devant le CC reste jusqu'au bout et réussit à créer les conditions d'un débat avec les autres participants. D'origine algérienne, il est âgé 20 ans et a suivi une formation courte de soudeur qui lui a permis de trouver un travail, ce qui ne l'empêche pas de continuer à fréquenter l'allée 1 où il retrouve ses amis. Consacré porte-parole du groupe à l'occasion de sa venue au CC, il profite de son intervention pour revenir à la charge sur le projet de local que la mairie refuse d'octroyer aux jeunes du quartier. Le compte rendu d'observation de la réunion donne un

\footnotetext{
${ }^{12}$ Ces deux citations sont extraites des carnets de terrain réalisés par les étudiants. Elles proviennent d'échanges en petits groupes visant à faire réagir les lycéens aux objectifs des CC.
} 
aperçu des conditions dans lesquelles s'effectue cette prise de parole et des échanges qu'elle suscite :

À la fin de la réunion, Mehdi est invité à prendre la parole et affirme que ce qu'il faudrait le plus pour les jeunes, c'est un local.

Pour le chef de projet politique de la ville, la question d'un local pour les jeunes est compliquée, mais Mehdi réagit en expliquant que de tels lieux existent ailleurs. Il cite Vaulx-en-Velin. Une habitante cite l'expérience de Vienne, soutenant l'idée que le CC peut s'inspirer de ce qui marche ailleurs. Mais le chef de projet avertit que personne ne mettra à disposition un local s'il n'est pas géré par une structure comme une association. Dans un esprit de conciliation, l'éducateur spécialisé propose alors de mettre à contribution le CC pour emmener un groupe de jeunes voir comment ça se passe à Vienne.

Mais Mehdi pointe un autre problème : pour que le local soit ouvert en soirée, l'idéal serait que ce soit un jeune du groupe qui gère le local. Il indique aussi qu'à son idée, le local devrait être destiné à tous les jeunes de Grigny, pas uniquement les jeunes du quartier. Les membres du conseil et Mehdi réfléchissent ensuite à l'idée d'ouvrir le local sur d'autres créneaux qu'en soirée, par exemple pour pouvoir travailler tranquillement. En fin de séance, l'éducateur invite Mehdi à réfléchir davantage au projet «pour être un peu plus crédible » et à « rassembler d'autres jeunes autour de cette idée ».

Par cette dernière consigne, le CC conditionne la participation de Mehdi à l'avancée du projet de local, alors même que ce dernier évoque en entretien les difficultés qu'il éprouve à mobiliser les jeunes du quartier sur ce projet. Bien qu'enthousiaste à la sortie du conseil, il ne donnera pas suite à cette première rencontre, malgré plusieurs relances de l'animatrice du centre social et des éducateurs présents sur le quartier. A Grigny, l'expérience participative se conclut donc à peu près de la même manière qu'à Saint-Fons et Vaulx-en-Velin, dans une sorte de confrontation paradoxale qui met en présence le public tant convoité des jeunes avec celui des conseillers citoyens en place (ou en train d'advenir dans le cas de Saint-Fons), mais sans vraiment leur permettre de se rencontrer, parce qu'elle maintient entre eux une certaine distance en réaffirmant les rapports de domination qui structurent classiquement les relations intergénérationnelles (Pitti, 2016). La répétition de ces confrontations paradoxales fait cependant question et nous amène, pour finir, à un certain nombre de constats sur la mise à contribution des jeunes dans les CC.

\section{Trois tentatives d'intégration, trois échecs, les mêmes ressorts d'exclusion}

Comment comprendre que dans chacune des situations observées, l'engouement du CC pour les jeunes se traduise au bout du compte par une incapacité à les intégrer, même lorsqu'ils se présentent avec l'envie de participer et de s'associer aux actions en cours ? Ce constat ressort en effet des trois études : à Grigny, la rencontre se solde par un début d'échange laissé sans suite par des conseillers en manque d'intérêt pour la question posée par les jeunes ; à Vaulxen-Velin, le bon accueil réservé aux lycéens et l'écoute attentive de leurs propositions se conclut par une invitation à revenir plus tard qui n'est pas entendue par les jeunes ; à SaintFons enfin, le désir de jeunes n'empêche pas de les marginaliser d'emblée et de les inciter à la fuite. Quelles sont précisément les causes de ces rendez-vous manqués ? Quels enseignements pouvons-nous en tirer à propos de la participation des jeunes aux CC ? 


\section{Une norme participative qui s'ajuste mal aux attentes des jeunes}

Ces rendez-vous manqués semblent d'abord liés au cadre extrêmement formel des trois instances, qui n'est pas de nature à favoriser l'engagement des jeunes. Cette rigidité, acceptée voire attendue par les membres institués des conseils citoyens, contraint les assemblées à un fonctionnement très normé qui ne convient pas toujours à la façon dont les jeunes voient leur participation. C'est le cas à Grigny, où les participants se réunissent une fois par mois, le lundi de $17 \mathrm{~h} 30$ à 19h30, dans une salle mise à disposition par la mairie, non loin de l'allée 1. Par cette régularité de fonctionnement, le $\mathrm{CC}$ tend alors à se confondre avec ce local fréquenté uniquement par les parents et les enfants du quartier, mais pas par les jeunes de l'allée 1 qui restent ainsi cantonnés à leur propre espace, au pied de l'immeuble voisin. Cette règle implicite, selon laquelle les réunions se tiennent normalement dans la salle réservée et pas « hors les murs », crée donc une sorte de barrière symbolique entre le territoire du CC et celui des jeunes qui, finalement, ne favorise pas la rencontre des deux groupes. De même, le déroulé des réunions, la mise en forme de comptes rendus écrits et le non-usage des outils de communication numériques opèrent comme autant d'obstacles qui n'incitent pas les jeunes à s'approprier les CC. Cette remarque est fréquemment revenue dans la bouche des lycéens de Vaulx-en-Velin et des jeunes de Saint-Fons.

Une autre difficulté vient de ce que les conseillers citoyens, par routine, manque de formation ou respect d'un minimum de formalité, attendent des publics convoités qu'ils viennent à eux et se conforment à leurs règles de fonctionnement. Dans les trois cas, les jeunes sont en effet contraints de se soumettre à l'ordre du jour et au format de la réunion, sans que la possibilité ne leur soit donnée d'émettre un avis sur le cadre et les conditions de leur prise de parole. La tentative d'intégration de Mehdi au CC de Grigny montre ainsi sa capacité à se conformer aux attentes en termes de ponctualité, de politesse et de qualité d'expression, mais aussi l'absence d'effort des conseillers qui lui font face pour adapter leur fonctionnement (en lui offrant par exemple la possibilité de réagir à l'ordre du jour) et se placer sur un pied d'égalité avec lui (en veillant à ce qu'il accède aux enjeux des autres sujets débattus pendant la réunion). Cette attitude des participants, également observée à Saint-Fons et Vaulx-en-Velin, rappelle que dans la confrontation entre les jeunes et leurs aînés, il est toujours question de division des pouvoirs : « les classifications par âge (mais aussi par sexe ou, bien sûr, par classe...) reviennent toujours à imposer des limites et à produire un ordre auquel chacun doit se tenir, dans lequel chacun doit se tenir à sa place » (Bourdieu, 1984 : 143-144). D'une certaine manière, les assemblées des $\mathrm{CC}$ tendent à la reproduction de cet ordre et en le perpétuant, annihilent les tentatives de participation des jeunes.

Sur un registre assez similaire, on note également une tendance manifeste à l'individualisation de l'exercice de la représentation, alors que les jeunes ont beaucoup de mal à envisager leur participation autrement qu'en groupe. Comme le note Cyprien Avenel à propos des jeunes hommes d'un quartier populaire de la périphérie de Bordeaux, « c'est par le biais du groupe, et l'appartenance à la cité, que l'individu a le sentiment d'exister socialement, alors que seul, il se sent méprisé, toujours menacé de déchoir» (Avenel, 2000 : 147). Ce constat semble également valoir dans le cadre restreint du CC, comme le montrent les lycéens de Vaulx-enVelin lorsqu'ils conditionnent leur participation à la possibilité de venir «en groupe », «à plusieurs » ou « avec un copain »". Ce comportement « grégaire », qui vise à se rassurer et se protéger, explique aussi les difficultés qu'ils rencontrent au moment de concrétiser leur

\footnotetext{
${ }^{13}$ Cette demande, qui n'est peut-être pas spécifique aux jeunes des quartiers populaires, est revenue à chaque fois que les étudiants ont invité les lycéens à préciser les conditions dans lesquelles ils pourraient participer au CC.
} 
engagement, aucun jeune n'osant vraiment prendre le risque de revenir seul au conseil pour en devenir membre à part entière sans les autres.

\section{Des échanges intimidants et un sentiment d'inutilité difficile à dissiper}

Si la norme participative des CC s'ajuste aussi mal aux dispositions des jeunes invités à s'intégrer à ces dispositifs, c'est aussi que les échanges avec les membres en place ont tendance à les maintenir dans une position d'infériorité en révélant un déficit manifeste de compétence. Cette difficulté concerne notamment la compréhension des enjeux associés aux sujets discutés, la connaissance des moyens d'action à disposition des participants et la maîtrise du langage expert utilisé par les acteurs de la politique de la ville présents aux réunions et certains habitants habitués au dialogue institutionnel. C'est ce que montre par exemple l'observation de la réunion du CC de Grigny où le porte-parole des jeunes de l'allée 1 fait face à une assemblée d'une dizaine de personnes constituée des quatre habitants réguliers, du chef de projet politique de la ville, de l'animatrice du centre social, de l'agent de développement local du bailleur, de la déléguée du préfet et de l'éducateur spécialisé qui l'accompagne à sa demande :

La réunion commence par un tour de table. Une habitante salue chaleureusement la présence de Mehdi en espérant que sa venue permettra d'impulser quelque chose « pour les jeunes » et « avec les jeunes ». Elle ajoute que cet objectif lui tient à cœur depuis la mise en place du CC. Les autres membres habitants confirment.

Une fois la réunion lancée, le chef de projet fait un point d'étape sur les programmes en cours de la politique de la ville et propose de former les conseillers sur ce sujet. Mehdi reste silencieux. Ensuite, deux membres du conseil font un retour sur leur participation aux rentrées citoyennes de Vaulx-en-Velin. L'habitante préoccupée du sort des jeunes interpelle à nouveau Mehdi : «Quelles sont les clés pour qu'on puisse justement les aborder, leur proposer de venir aux réunions et leur dire qu'on va les écouter ?» Elle tient notamment à trouver un sujet qui intéresse les jeunes. Mehdi répond qu'il y a beaucoup d'outils pour les jeunes mais que selon lui, personne n'est vraiment au courant. Il évoque aussi la question du local sur laquelle il reviendra à la fin de la réunion.

Poursuivant sur la question des jeunes, le chef de projet détaille la procédure à suivre pour programmer une action pour les jeunes et alerte sur les budgets limités des partenaires. L'éducateur spécialisé réagit : « Je ne pense pas que ça doit coûter beaucoup ce genre d'action ». Le chef de projet répond que les multiples actions font au final des montants importants. Mehdi, qui ne semble pas avoir saisi le sens de cet échange, ne répond rien. Le chef de projet l'interpelle alors sur la nécessité de mieux informer les jeunes des actions existantes : "Moi je retiens ce que disait Mehdi toute à l'heure, c'est qu'apparemment il y a pas mal de choses qui se passent sur Grigny dont personne n'est au courant. L'idée c'est qu'on puisse faire ce travail ensemble, avec toi, d'autres jeunes, etc., qu'on puisse recenser un peu tout ça, quitte à ce qu'on fasse une plaquette un peu simple. L'idée, ça serait de rapprocher les outils qui existent des jeunes que ça doit intéresser. » La proposition n'est cependant pas bien comprise par Mehdi qui répond : «Moi, franchement en venant ici, j'ai appris plein de choses. Il y a plein de choses que je ne savais pas qu'on avait ça en gros. »

La réunion se poursuit et il faut attendre une heure et demie d'échanges pour que Mehdi soit enfin invité à prendre la parole au nom des jeunes du quartier sur la question du local.

Mehdi est ainsi cantonné durant toute la réunion dans une position d'apprenant qui ne lui permet pas de prendre sa place dans un débat mené de bout en bout par les acteurs institutionnels membres du CC. Cette difficulté, propre à l'engagement des «publics profanes » dans les arènes fortement technicisées (Callon et al., 2001), joue ici clairement contre l'intégration du jeune homme dans l'assemblée. Sa méconnaissance des sigles et des acronymes, des dispositifs légaux de la politique de la ville, mais également des conditions 
normales d'attribution d'un local municipal, le desservent en l'excluant peu à peu de la discussion et en autorisant ses interlocuteurs, au terme de la réunion, à lui proposer de retravailler le contenu du projet « pour être un peu plus crédible ».

Enfin, une dernière complication observée vient de la difficulté à faire correspondre le temps institutionnel du CC avec le temps de vie des jeunes sollicités qui, plus que les autres, inscrivent leurs demandes dans l'immédiateté et l'urgence (Hbila, 2012). A Vaulx-en-Velin par exemple, les lycéens n'adhèrent pas du tout à l'idée d'un engagement qui suppose de se projeter sur le moyen et/ou long terme en s'astreignant à une certaine assiduité pour des résultats a priori incertains. Le seul horizon qu'ils envisagent concrètement est celui de la réunion à laquelle ils sont conviés, pour des résultats qu'ils pensent instantanés. Leurs échéances ne vont pas audelà. De même, le porte-parole des jeunes de Grigny privilégie dans son action une efficacité immédiate et n'imagine pas, en se présentant devant le CC, repartir avec un ensemble d'exigences à satisfaire (mobiliser ses pairs, créer une structure associative, préciser le fonctionnement du local, etc.) pour espérer soumettre à nouveau son projet. En retour, l'absence de réponse provoque frustration et résignation, et ce d'autant que cette expérience vécue négativement s'ajoute à une longue liste de refus préalables, de non réponses et de demandes non satisfaites. C'est ce qu'explique cet autre jeune de l'allée 1 :

«Avant on voyait par le quartier... Je te dis "on", parce qu'on est plusieurs à être comme ça aujourd'hui, tu vois... Avant, on était plus investi dans le fait d'aller avec les éducs, d'aller au PIJ [point information jeunesse]... On était plus investi dans ces trucs-là. Bon et puis du jour au lendemain on s'en foutait quoi ! En fait il n'y avait plus rien à faire! Parce ce qu'on a bien vu que pendant 5-10 ans, il n'y avait pas eu de changement sur la ville. Il n'y a jamais eu de changement. Quand il y a du changement, c'est pour les autres, ce n'est pas pour les jeunes. Tu vois, pour moi c'est de la discrimination. Tu vois ? Parce que ça fait hyper longtemps, ça fait super longtemps qu'on demande des gradins au stade, des vestiaires, un local... » (entretien)

Finalement, l'analyse comparée des trois tentatives d'intégration orchestrée par les étudiants permet d'isoler plusieurs facteurs explicatifs du désengagement des jeunes sollicités par les CC de Grigny, Saint-Fons et Vaulx-en-Velin : la formalité des échanges qui les conforte dans l'idée qu'ils ne sont pas à leur place dans ce type d'assemblée et les réinstallent dans un mode de soumission aux adultes qui ne leur convient pas ; l'individualisation de la représentation qui contrarie leur conception de la participation comme activité collective; l'image d'incompétence que leur renvoient les autres participants en ne faisant pas l'effort de se mettre à niveau ; et enfin leur désir d'immédiateté qui s'accommode très mal du temps long des actions et réflexions engagées par les membres de chaque assemblée. Ces différents ressorts d'exclusion, qui jouent différemment dans chaque situation observée, ont alors tendance à se combiner pour compromettre l'intégration des jeunes qui s'étaient pourtant montrés intéressés pour endosser un rôle de conseiller citoyen.

\section{Conclusion}

Chacun à leur façon, les CC de Grigny, Saint-Fons et Vaulx-en-Velin montrent une volonté forte de dépasser les limites fréquemment rencontrées par les instances de participation. Dans les trois cas, l'absence de jeunes est déplorée et leur participation recherchée, soit pour tenter de résoudre avec eux certains problèmes du quartier, soit parce que leur présence est considérée comme un gage de représentativité de nature à renforcer la légitimité de l'instance. Pourtant, malgré l'envie manifeste de capter ce public spécifique et de l'intéresser aux enjeux de la représentation, les conseillers citoyens observés de part et d'autre n'ont pas su éviter les écueils classiques de la participation des jeunes. Le travail effectué par les étudiants avait pourtant permis de faire émerger les conditions d'un échange sur les questions de la participation 
citoyenne et d'instaurer un lien de confiance en vue d'une possible intégration aux dispositifs existants. Mais devant la réalité du CC et de son fonctionnement, les jeunes ont à chaque fois choisi de ne pas donner suite au premier pas effectué. Plusieurs explications sont alors ressorties de nos observations. La première et peut-être la plus significative résulte du formalisme de l'instance qui engendre un climat peu propice à la prise de parole des adolescents et jeunes adultes de ces quartiers populaires, surtout quand ils se trouvent dominés en nombre et contraints de se conformer à des codes qu'ils ne maitrisent pas. Mais l'individualisation de l'exercice de représentation, l'ordre institué entre les différentes classes d'âge, le déficit de connaissances techniques et l'incompatibilité du temps institutionnel avec leur désir d'immédiateté sont également des caractéristiques qui, dans les trois cas, ont pu contribuer à décourager la participation des publics considérés.

Si la chronique de ces rendez-vous manqués permet de montrer comment les trois dispositifs étudiés reproduisent malgré eux les mécanismes qui détournent habituellement les jeunes des dispositifs de participation institutionnalisés, il ne faudrait cependant pas conclure que tous les $\mathrm{CC}$ fonctionnent comme des machines à exclure les jeunes ou, dans une formulation moins radicale, que le CC, tel qu'il a été institué par la loi Lamy de 2014, n'est pas adapté à la participation des jeunes des quartiers populaires. Plusieurs limites ressortent en effet de nos analyses qui empêchent cette montée en généralité. La première est que nos observations restent focalisées sur trois cas de l'agglomération lyonnaise qui ne sont pas forcément représentatifs de la diversité des $\mathrm{CC}$ créés ces dernières années en France. La deuxième vient de notre dispositif d'enquête qui ne permet pas de caractériser finement les groupes de jeunes considérés et dans ces conditions, d'identifier précisément les propriétés sociales qui conditionnent leur expérience du CC. Une troisième limite vient enfin de notre objet qui réduit la focale aux jeunes des quartiers populaires et n'aide pas à cerner ce qui leur est spécifique dans le rapport à la participation institutionnalisée, en comparaison avec d'autres publics de jeunes notamment.

En dépit de cette invitation à la prudence dans la mise en forme de nos résultats, il reste malgré tout que la norme participative associée à ces trois CC échoue dans son projet de réaffiliation des jeunes des quartiers populaires, de façon assez comparable à tous ces autres dispositifs qui depuis de nombreuses années ne mobilisent que parmi les groupes déjà affiliés socialement et économiquement (Bacqué, Sintomer, 2001). Doit-on pour autant en conclure à l'impossibilité de la participation des jeunes des quartiers populaires dans un cadre institutionnalisé comme peut l'être celui des trois CC étudiés ? Assurément oui si la norme participative ne se détache pas du formalisme et de la dépendance aux institutions locales (mairies, centres sociaux, associations de soutien à la citoyenneté, etc.) que l'on a observés ici. Mais sans doute faut-il aussi compter sur la capacité de ces instances à sortir des cadres dans lesquels la participation est habituellement pensée et mise en œuvre. Car la vision fataliste de l'impossible participation des jeunes des quartiers populaires est sans rapport avec l'engagement dont ces derniers peuvent témoigner dans d'autres situations (Becquet, de Linares, 2005). C'est ce que montrent par exemple toutes les actions qui visent justement à permettre l'expression de cette conscience politique. À cet égard, le projet PoliCité, recherche-action menée par un groupe d'adolescents d'un centre social de Vaulx-en-Velin ayant pour objectif d'améliorer les relations policepopulation, constitue un bon contre-exemple à la vision fataliste précédemment mentionnée (Purenne, 2018). Offrant des espaces pour dire et comprendre la violence des interactions qu'ils vivent au quotidien ou l'injustice des situations auxquelles ils sont régulièrement confrontés, le projet mobilise les jeunes depuis maintenant plus d'un an et demi, montrant au passage que leur engagement peut être important lorsque la forme de la participation et les thématiques abordées leur correspondent. Autrement dit, ce type d'expérience réussit précisément là où les 
trois CC ont échoué, c'est-à-dire dans leur capacité à être un espace de socialisation politique permettant aux jeunes de s'exprimer librement et de monter en généralité.

\section{Bibliographie}

Aquatias S., 1997, "Jeunes de banlieue, entre communauté et société. Une approche socio-anthropologique $\quad \mathrm{du} \quad \mathrm{lien} \quad$ social », Socio-anthropologie, 2, http://journals.openedition .org/socio-anthropologie/34.

Avenel C., 2004, Sociologie des « quartiers sensibles », Paris, Armand Colin.

Avenel C., 2000, «Les jeunes hommes et le territoire dans un quartier de grands ensembles », Lien social et Politiques, 43, p. 143-154.

Bacqué M.-H., Mechmache M., 2013, Pour une réforme radicale de la politique de la ville, Rapport au ministre délégué chargé de la ville, Le pouvoir d'Agir.

Bacqué M.-H., Sintomer Y., 2001, «Affiliations et désaffiliations en banlieue. Réflexions à partir des exemples de Saint-Denis et d'Aubervilliers », Revue française de sociologie, 42(2), p. 217-249.

Bataille J.-M., 2013, « Jeunes et participation, mise en perspective historique et interrogation sur les pratiques contemporaines », in GIS Démocratie et Participation, Actes des 3èmesjournées doctorales sur la participation et la démocratie participative, http://www.participation-et-democratie.fr/fr/node/1625.

Becquet V., 2006, « Participation des jeunes : regards sur six pays », Agora débats/jeunesses, 42, p. 14-29.

Becquet V., de Linares C. (dir.), 2005, Quand les jeunes s'engagent. Entre expérimentations et constructions identitaires, Paris, L'Harmattan-INJEP.

Bier B., 2009, «Jeunes de quartier populaire et institutions », Textes de l'INJEP, http://www.ressourcesjeunesse.fr/IMG/pdf/jeunes institutions bier.pdf.

Bordes V., Vulbeau A., 2004, L'alternative jeunesse, Paris, Édition de l'Atelier.

Bordet J., 2007, Oui à une société avec les jeunes des cités! Sortir de la spirale sécuritaire, Paris, Les Éditions de l'Atelier.

Bourdieu P., 1984, Questions de sociologie, Paris, Éditions de Minuit.

Callon M., Lascoumes P., Barthe Y., 2001, Agir dans un monde incertain. Essai sur la démocratie technique, Paris, Éditions du Seuil.

Castel R., 2006, «La discrimination négative. Le déficit de citoyenneté des jeunes de banlieue », Annales. Histoire, Sciences Sociales, 61(4), p. 777-808.

Cortéséro R., 2010, «Entre l'émeute et le ghetto. Quels cadres de socialisation politique pour les jeunes des banlieues populaires ? », Education et sociétés, 25, p. 65-81.

Daquin A., 2017a, Jeunes et conseil citoyen : quelles opportunités de renouvellement de la participation dans les quartiers populaires? Le cas de Grigny (69), Rapport d'étude, Programme de co-évaluation nationale des conseils citoyens, Université Paris Nanterre.

Daquin A., 2017b, La construction du problème des « jeunes de quartier ». Étude du rapport entre jeunes adultes et acteurs locaux d'un quartier en politique de la ville, Mémoire de master 2, Universités de Lyon et Jean Monnet Saint-Étienne, IEP de Lyon.

Derville G., 1997, «La stigmatisation des jeunes de banlieue », Communication et langages, 113, p. 104-117. 
Estèbe P., 2001, «La politique de la ville et la jeunesse », Agora débats/jeunesses, 5(1), p. 3138 .

Galland O., 2009, Les jeunes, Paris, La Découverte.

Guénolé T., 2015, Les jeunes de banlieue mangent-ils les enfants?, Lormont, Le Bord de l'eau. Hadj Belgacem S., 2016, « Reconquérir la jeunesse des cités ? Enjeux et contradictions d'un Conseil Local des Jeunes dans une municipalité communiste », Mouvements, 85, p. 95-103.

Hamidi C., 2006, «Éléments pour une approche interactionniste de la politisation. Engagement associatif et rapport au politique dans des associations locales issues de l'immigration », Revue française de science politique, 56(1), p. 5-25.

Hbila C., 2014, «La participation des jeunes des quartiers populaires : un engagement autre malgré des freins », Sociétés et jeunesses en difficulté, 14, http://sejed.revues.org/7608.

Hbila C., 2012, Jeunes de quartiers populaires et politiques de jeunesse. Adhésion et résistance des jeunes, Thèse de Sociologie, Université Rennes 2.

Huet M., 2018, Les pratiques urbaines des lycéens de quartiers populaires : quelles expériences de l'altérité ?, Thèse de Géographie, Aménagement et Urbanisme, Université de Lyon.

INJEP, 2012, « Jeunes des quartiers populaires », Les fiches repères de l'observatoire de la jeunesse et des politiques de jeunesse, décembre 2012, p. 1-4.

Koebel M., 2001, «La préoccupation de la jeunesse comme ressource politique », in A. Vulbeau (dir.), La jeunesse comme ressource. Expérimentations et expérience dans l'espace public, Paris, Eres, p. 9-16.

Lapeyronnie D., 1987, « Assimilation, mobilisation et action collective chez les jeunes de la seconde génération de l'immigration maghrébine », Revue Française de Sociologie, 28(2), p. 287-318.

Lepoutre D., 1997, Cæur de banlieue. Codes, rites et langages, Paris, Éditions Odile Jacob.

Loncle P., 2008, Pourquoi faire participer les jeunes? Expériences locales en Europe, Paris, L'Harmattan.

Marlière E., 2008, «Les "jeunes de cité". Territoires et pratiques culturelles », Ethnologie française, 38(4), p. 711-721.

Mazeaud A., 2012, «Allocation de l'argent public et budget participatif des lycées : règles du jeu et pratiques délibératives », Genèses, 88, p. 89-113.

Mucchielli L., 1999, «Violences urbaines, réactions collectives et représentations de classe chez les jeunes des quartiers relégués de la France des années 1990 », Actuel Marx, 26, p. 85118.

Pitti I., 2016, «Participation civique et politique des jeunes: rôle des relations intergénérationnelles », Agora débats/jeunesses, 73, p. 21-34.

Purenne A., 2018, «Comment faire de la sociologie avec des jeunes habitants de quartier populaire ?», in Laforgue D. (dir.), Le faire sociologique. Épreuves et horizons d'une recherche impliquée, Chambéry, Université Savoie Mont-Blanc, LLSTEI, p. 155-182.

Tourette F., 2006, « Jeunesse et politique de la ville : quels jeunes pour quelles politiques ? », Siècles, 24, p. 49-74.

Tourrilhes C., 2006, «Construction sociale d'une jeunesse en difficulté », Agora débats/jeunesses, 40, p. 108-119.

Truong F., 2015, Jeunesses françaises. Bac + 5 made in banlieue, Paris, La Découverte. 
Vulbeau Alain (dir.), 2001, La jeunesse comme ressource. Expérimentations et expériences dans l'espace public, Paris, Érès. 